\title{
Infratentorial subdural empyema associated with long standing occipital dermoid sinus: case report
}

\begin{abstract}
Infratentorial subdural empyema is a rare form of life threatening intracranial infection, requiring immediate neurosurgical intervention.

We present this 4-years-old girl with posterior fossa subdural empyema which is associated with congenital occipital dermal sinus. A contrast-enhanced CT scan showed an infratentorial supracerebellar hypodense fluid collection with the peripheral rim enhancement to the left of the midline that raised suspicion of a subdural empyema with supratentorial mild ventricular dilatation which was confirmed by MRI with contrast. The patient was operated through sub-occipital decompression and drainage of the collection and the samples was sent for culture and sensitivity. Dermal sinus can be a cause for intracranial infection and should be investigated to rule out intradural connection. Infratentorial subdural empyema should be managed urgently by neurosurgical intervention to prevent further life threatening complications.
\end{abstract}

Keywords: Infratentorial, Subdural empyema, Dermal sinus, Craniectomy, Hydrocephalus
Volume I Issue 5 - 2014

\author{
Mohamed MF Okasha,' Ahmed Beheiry, ${ }^{2}$ \\ Yasser M Elkhwalka² \\ 'Department of Neurosurgery, Newcastle upon Tyne Hospitals, \\ United Kingdom \\ ${ }^{2}$ Department of Neurosurgery, Damanhour National Medical \\ Institute, Egypt
}

\begin{abstract}
Correspondence: Mohamed MF Okasha, Neurosurgery Department, Royal Victoria Infirmary, Newcastle upon Tyne Hospitals, NHS foundation Trusts, NEI 4LP, United Kingdom, Tel 447784668332,Email okasha_neuro@yahoo.com
\end{abstract}

Received: September 04, 2014 | Published: September 22, 2014
Abbreviations: CT, Computed Tomography; MRI, Magnetic Resonance Imaging; CNS, Central Nervous System; CSF, Cerebrospinal Fluid; GCS, Glasgow Coma Scale; CVP, Central Venous Pressure; EVD, External Ventricular Drain

\section{Introduction}

Subdural empyema is defined as a collection of pus in the preformed space between the cranial dura mater and arachnoid mater. ${ }^{1}$ Due to its life threatening nature, most cases require neurosurgical drainage of the collection. Infratentorial subdural empyemas are uncommon constituting only $0.6 \%$ of all cases of intracranial suppurative conditions. ${ }^{2}$ A congenital dermal sinus is a tract lined by epidermis communicting between the skin and the deeper tissues and may be connected with the central nervous system. It is rarely connected with posterior fossa. ${ }^{3}$

In this paper, we report a rare case of congenital dermal sinus with acute presentation of subtentorial subdural empyema which was successfully treated with neurosurgical intervention.

\section{Case presentation}

A four years old girl known to have a long standing congenital midline occipital scalp dimple (Figure 1) which was treated expectantly as a superficial dermal sinus. She presented over 10 days period by persistent progressive pyrexia and headache, for which she was admitted in one of the local community hospital for suspected bacteremia versus meningitis. Routine blood tests revealed polymorphic leukocytosis, elevated erythrocyte sedimentation rate and C Reactive protein. In addition CSF analysis raised the suspicion of intracranial infection. She was initially treated with antibiotics (Penicillin, Ceftriaxone and Metronidazole) for a week prior to diagnosis. After a week she developed headache and deterioration of conscious level. We received a referral about her condition and arranged for her CT and MRI with contrast (Figure 2 and 3). She was transferred to our care in Damanhour Teaching Hospital for investigation and management, with GCS of 13/15, photophobia, mild cerebellar signs, neck stiffness and two discharging midline occipital dermal sinuses. CT and MRI were positive for infratentorial supracerebellar hypodense collection with contrast enhancement suggestive for empyema.

The patient's condition required emergency surgery on day 10 of presentation involving a sub-occipital decompressive craniectomy and drainage of empyema. We started the procedure with insertion of external ventricular drain through occipital burr-hole. Midline suboccipital incision was performed; excision of the dermal sinus tract was then carried out. The dermal sinus was connected by a tract extending from scalp to the dura through a tiny midline occipital defect. Suboccipital decompressive craniectomy was done followed by opening of the dura as Y shaped and drainage of purulent collection which was sampled for culture and sensitivity. The wound was irrigated with warm saline and vancomycin. Closure in layers with three-way CVP catheter for drainage and further irrigation.

Postoperative care in the ICU was uneventful with improvement of the conscious level and reduced severity of headache. External ventricular drain was set to $10 \mathrm{~cm} \mathrm{H2O}$. It was clamped for 24 hour and was removed on day 4 postoperatively. We continued the empirical antibiotics until the results of culture and sensitivity which showed Staphelococcus aureus and sensitive to the same antibiotics. The patient gradually returned back to normal activity with mild headache and cerebellar ataxia which had improved a few weeks later.

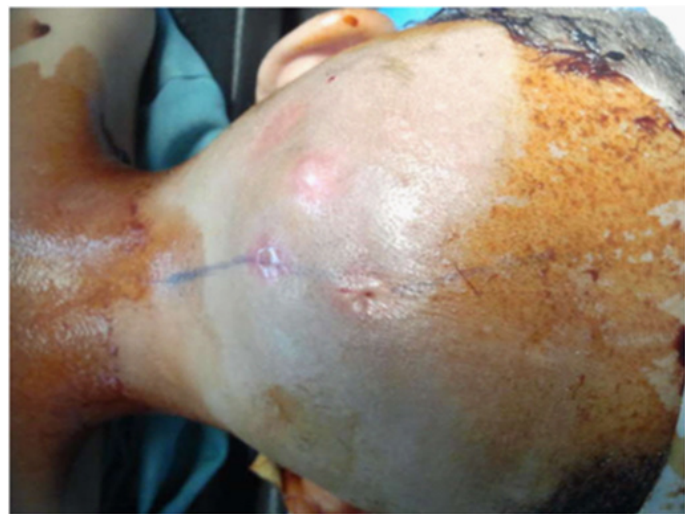

Figure I Occipital midline congenital dermal sinus. 


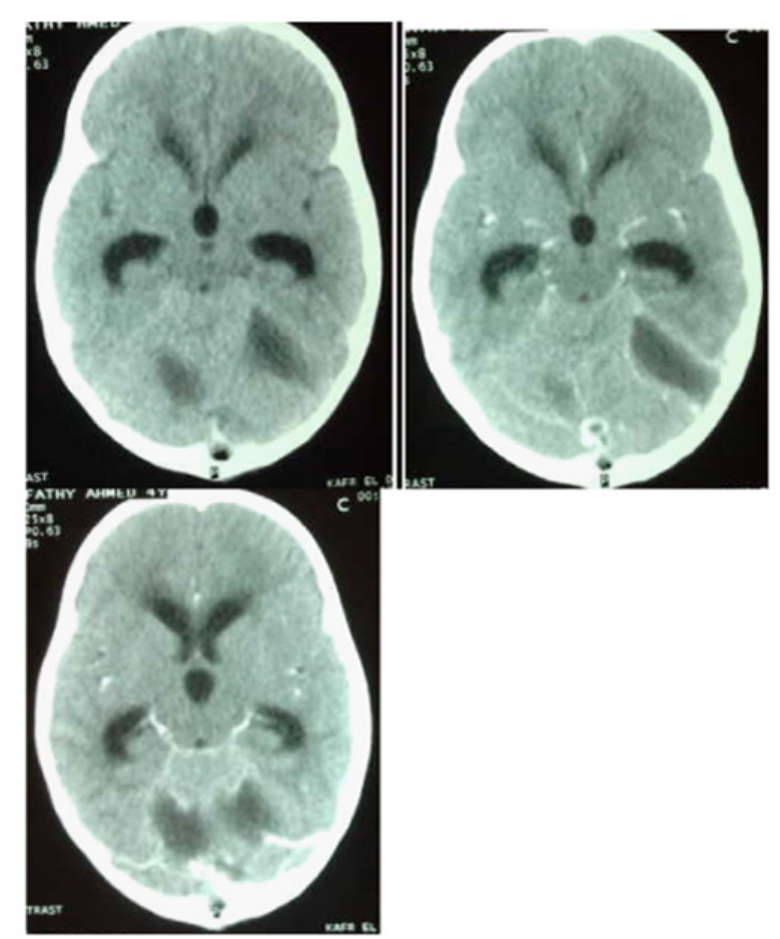

Figure 2 Pre and post contrast CT head demonstrating infratentorial hypodense collection with contrast enhancement, hydrocephalic changes also can be noticed.

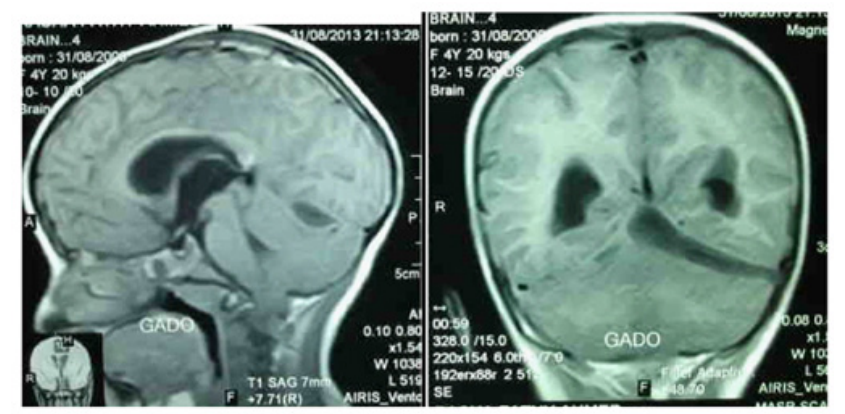

Figure 3 MRI Head post-contrast showing enhancing collection.

\section{Discussion}

Our case represents a very rare condition with infratentorial subdural empyema caused by dermal sinus connected to the subdural space. This condition is associated with significant morbidity and mortality with only few reported cases in the literatures describing the posterior fossa subdural empyema. ${ }^{4} \mathrm{CNS}$ infection with dermal sinus also is an extremely uncommon presentation. ${ }^{5} \mathrm{MRI}$ and DWI are the preferred imaging modality for subdural Empyema. ${ }^{6}$
Hydrocephalus is a common complication of this condition which mostly requires external ventricular drainage during surgery or postoperatively while Shunt placement was required in some cases. ${ }^{7}$

Emergent surgical evacuation, simultaneous management of the primary source of infection and intravenous long course of appropriate antibiotics are all recommended. ${ }^{8}$

The diagnosis and management in our case was delayed because of delayed presentation of the intracranial suppuration and hydrocephalus, however surgical evacuation, ventricular drainage and antibiotic administration improved the condition and reduced the risk of deterioration of the condition and the major threatening complications.

\section{Conclusion}

In conclusion, infratentorial subdural empyema is an uncommon life threatening condition can be rarely caused by dermal sinus connected to the CNS. Diagnosis is mainly by MRI and emergency drainage of the collection is mandatory to prevent serious life threatening complications. Hydrocephalus is a common complication of this disease, requiring surgical diversion of the CSF either by EVD or permanently by shunt insertion after resolution of the suppuration.

\section{Acknowledgments}

None.

\section{Conflicts of interest}

None.

\section{References}

1. Tsai YD, Chang WN, She CC, et al. Intracranial suppuration: A clinical comparison of subdural empyemas and epidural abscesses. Surg Neurol. 2003;59(3):191-196.

2. Nathoo N, Nadvi SS, van Dellen JR. Infratentorial empyema: analysis of 22 cases. Neurosurgery. 1997;41(6):1263-1268.

3. Higashi S, Takinami K, Yamashita J. Occipital dermal sinus associated with dermoid cyst in the fourth ventricle. AJNR Am J Neuroradiol. 1995;16(4 Suppl):945-948.

4. van de Beek D, Campeau NG, Wijdicks EF. The clinical challenge of recognizing infratentorial empyema. Neurology. 2007;69(5):477-481.

5. Kanev PM, Salazar JC. Unusual CNS infection from a subtorcular dermal sinus. Acta Paediatr. 2010;99(4):627-629.

6. Vazquez E, Castellote A, Piqueras J, et al. Imaging of complications of acute mastoiditis in children. Radio Graphics. 2003;23(2):359-372.

7. Venkatesh M, Pandey P, Devi BI, et al. Pediatric infratentorial subdural empyema: analysis of 4 cases. J Neurosurg. 2006;105(5 Suppl).370-377.

8. Kojima A, Yamaguchi N, Okui S. Supra- and infratentorial subdural empyema secondary to septicemia in a patient with liver abscess — case report. Neurol Med Chir (Tokyo). 2004;44(2):90-93. 